\title{
CHANGES IN PLASMA SPHINGOLIPID LEVELS AGAINST THE BACKGROUND OF LIPID-LOWERING THERAPY IN PATIENTS WITH PREMATURE ATHEROSCLEROSIS
}

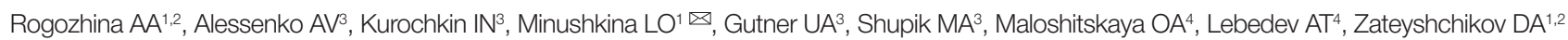 \\ ${ }^{1}$ Central State Medical Academy of Department of Presidential Affairs, Moscow, Russia \\ ${ }^{2}$ City Clinical Hospital № 51, Moscow, Russia \\ ${ }^{3}$ Institute of Biochemical Physics named after N. M. Emanuel, Moscow, Russia \\ ${ }^{4}$ Lomonosov Moscow State University, Moscow, Russia
}

\begin{abstract}
Lipid-lowering drugs affect standard lipoproteins. However, we have no knowledge of changes in other plasma lipids upon treatment. The study was aimed to assess the dynamic changes in cholesterol, high- and low-density lipoproteins (HDL and LDL), triglycerides, and sphingolipids against the background of lipidlowering therapy in patients with premature coronary artery disease, atherosclerosis and hypercholesterolemia. A total of 18 patients were enrolled (the average age was $53 \pm 6.7$ years): in group 1 , six patients received starting statin doses; group 2 included six patients, who failed to achieve LDL target levels against the background of treatment with starting statin doses, and received escalated statin doses; seven patients in group 3 failed to achieve LDL target levels against the background of treatment with maximum tolerated doses of statins and ezetimibe, and received alirocumab. Sphingolipid levels were assessed by mass spectrometry. In group 1, the decreased levels of ceramide Cer 14:1 $(p=0.046)$ and sphingomyelins SM 22:1, SM 22:0, SM 24:0 ( $p=0.028)$ were observed. There were no significant changes in the levels of total cholesterol, LDL-C, HDL-C, and triglycerides. In group 2, the significantly decreased levels of total cholesterol $(p=0.028)$, LDL $(p=0.043)$, sphingomyelins SM 18:1, SM 24:1 and SM 26:1, and ceramide Cer 16:1 ( $p=0.028)$ were observed. The level of Cer 22:1 significantly increased $(p=0.028)$. In group 3, total cholesterol decreased by $36.2 \%$, and LDL-C $(p=0.018)$ decreased by $60.1 \%$ compared to baseline $(\triangle \mathrm{LDL}-\mathrm{C}=-2.67$ $\pm 3.12)$; the elevated levels of ceramide Cer 22:1 $(p=0.028)$ were observed. It has been shown, that decreased sphingomyelin levels are associated with statin therapy and correlate with decreased levels of LDL-C. No significant dynamic changes in ceramides and ceramide risk against the background of statin therapy were observed, however, PCSK9 inhibitor added to therapy reduced the Cer 16:0/24:0 ratio.
\end{abstract}

Keywords: atherosclerosis, sphingomyelins, sphingosine, ceramides, lipid-lowering therapy

Funding: RFBR grant 19-04-00870A, Sphingolipidome Analysis of Cardiovascular Disease Markers.

Author contribution: Rogozhina AA — sampling, data acquisition; Alessenko AV — project management; Kurochkin IN — data analysis; Minushkina LO — data analysis, manuscript writing; Gutner UA, Shupik MA, Maloshitskaya OA — sample preparation, laboratory tests, data analysis; Lebedev AT, Zateyshchikov DA — study planning, data analysis, manuscript editing.

Compliance with ethical standards: the study was approved by the Ethics Committee of City Clinical Hospital № 51, Moscow (protocol № 02/19 dated February 7, 2019). Informed consent was submitted by all patients.

$\bowtie$ Correspondence should be addressed: Larisa O. Minushkina Marshala Timoshenko, 19, str. 1A, Moscow, 121359; minushkina@mail.ru

Received: 24.05.2021 Accepted: 13.06.2021 Published online: 23.06.2021

DOI: $10.24075 /$ brsmu.2021.030

\section{ИЗМЕНЕНИЕ КОНЦЕНТРАЦИИ СФИНГОЛИПИДОВ В ПЛАЗМЕ КРОВИ НА ФОНЕ ГИПОЛИПИДЕМИЧЕСКОЙ ТЕРАПИИ У ПАЦИЕНТОВ С РАННИМ АТЕРОСКЛЕРОЗОМ}

А. А. Рогожина ${ }^{1,2}$, А. В. Алесенко, И. Н. Курочкин ${ }^{3}$, Л. О. Минушкина ${ }^{1}$, У. А. Гутнер ${ }^{3}$, М. А. Шупик , О. А. Малошицкая ${ }^{4}$, А. Т. Лебедев Д. А. Затейщиков ${ }^{1,2}$

${ }^{1}$ Центральная государственная медицинская академия управления делами президента РФ, Москва, Россия

2 Городская клиническая больница № 51, Москва, Россия

${ }^{3}$ Институт биохимической физики имени Н. М. Эмануэля, Москва, Россия

${ }^{4}$ Московский государственный университет имени М. В. Ломоносова, Москва, Россия

\begin{abstract}
Гиполипидемические препараты влияют на стандартные липопротеины, но как изменяются при лечении другие липиды плазмы - не известно. Целью работы было оценить динамику холестерина (ХС), липопротеинов высокой и низкой плотности (ЛВП и ЛНП), триглицеридов (ТГ) и сфинголипидов у пациентов с преждевременно развившейся ишемической болезнью сердца, атеросклерозом и гиперхолистеринемией на фоне гиполипидемической терапии. В исследование включено 18 больных (средний возраст 53 土 6,7 года): в 1-й группе 6 пациентов получили стартовые дозы статинов; во 2-ю группу вошли 6 пациентов, не достигших на фоне стартовой терапии статинами целевых уровней ЛНП и принимавших увеличенные дозы статинов; 7 пациентов 3-й группы не достигли целевых уровней ЛНП на фоне терапии максимально переносимыми дозами статинов и эзетимиба и получали алирокумаб. Исследование уровня сфинголипидов проводили методом масс-спектрометрии. В 1-й группе отмечено снижение уровня церамида Сег 14:1 ( $p=0,046)$ и сфингомиелинов SM 22:1, SM 22:0, SM 24:0 ( $p=0,028)$. Уровни общего ХС (ОХС), ХС ЛНП, ХС ЛВП и ТГ существенно не изменились. Во 2-й группе отмечено достоверное снижение уровня ОХС ( $p=0,028)$, ЛНП ( $p=0,043)$, ссингомиелинов SM 18:1, SM 24:1 и SM 26:1, церамида Сеr 16:1 ( $p=0,028)$. Cer $22: 1$ достоверно увеличился $(p=0,028)$. В 3-й группе ОХС снизился на $36,2 \%, X C$ ЛНП $(p=0,018)-$ на $60,1 \%$ от исходных значений $(\triangle X C$ ЛНП = -2,67 $\pm 3,12)$, выявлено повышение уровня церамида Cer 22:1 ( $p=0,028)$. Показано, что снижение содержания сфингомиелинов происходит на фоне терапии статинами и коррелирует со снижением уровня ХС ЛНП. Значимой динамики церамидов и церамидного риска на фоне терапии статинами не зарегистрировано, однако при добавлении к терапии ингибитора PCSK9 произошло уменьшение соотношения Сеr 16:0/24:0.
\end{abstract}

Ключевые слова: атеросклероз, сфингомиелины, сфингозин, церамиды, гиполипидемическая терапия

Финансирование: грант РФФИ 19-04-00870А «Сфинголипидомный анализ маркеров сердечно-сосудистых заболеваний».

Вклад авторов: А. А. Рогожина - отбор материала, сбор данных; А. В. Алесенко - руководитель проекта; И. Н. Курочкин - анализ данных; Л. О. Минушкина - анализ данных, написание статьи; У. А. Гутнер, М. А. Шупик, О. А. Малошицкая - пробоподготовка, проведение лабораторных исследований, анализ данных; А. Т. Лебедев, Д. А. Затейщиков - планирование работы, анализ данных, редактирование статьи.

Соблюдение этических стандартов: исследование одобрено этическим комитетом Городской клинической больницы № 51 г. Москва (протокол № 02/19 от 7 февраля 2019 г.). Все пациенты подписали добровольное инсормированное согласие на участие в исследовании.

$\bigotimes$ Для корреспонденции: Лариса Олеговна Минушкина ул. М. Тимошенко, д. 19, стр. 1А, г. Москва, 121359; minushkina@mail.ru

Статья получена: 24.05.2021 Статья принята к печати: 13.06.2021 Опубликована онлайн: 23.06.2021

DOI: $10.24075 /$ vrgmu.2021.030 
In connection with increasing disability and mortality due to coronary artery disease (CAD) among young people [1], as well as with emergence of novel diagnostic methods, the in-depth investigation of risk factors, prevention methods, and complications of premature CAD (i. e. with the onset before the age of 55 years in men, and before the age of 60 years in women) [2], becomes more and more urgent.

The most significant risk factor for atherosclerosis is dyslipidemia, in particular, the elevated levels of lowdensity lipoproteins (LDL-C). These are directly associated with increased risk of cardiovascular disease [3]; hypercholesterolemia exceeding $4.9 \mathrm{mmol} / \mathrm{L}$ increases the risk of CAD by six times [4].

Due to developing mass spectrometry methods, the new laboratory parameters have been increasingly determined as additional markers of cardiovascular risk in patients with CAD, among them sphingolipids.

Sphingolipids contain the molecules of organic aliphatic amino alcohol sphingosine. It has been found, that sphingolipid catabolism is associated with cholesterol catabolism [5]. Cholesterol content of the membranes strongly correlates with the sphingolipid content (ceramides, sphingosines, and sphingosine-1-phosphate). Sphingomyelins form the myelin sheath cell membranes, and are detected in the atherosclerotic plagues [6]; ceramides contribute to cell proliferation, apoptosis and inflammation associated with atherosclerosis [7]. Currently, there is some evidence that ceramide levels, especially the levels of Cer16:0, correlate with the risk of atherosclerosis complications. These data served as the basis for calculation of the specific ceramide risk score (CRS) [8].

All the patients at high and extremely high risk for atherosclerosis have indications for high-intensity lipid-lowering therapy aimed to correct dyslipidemia and to reduce the risk of atherosclerosis progression.

The study was aimed to assess the dynamic changes in standard lipid indicators and sphingolipids against the background of lipid-lowering therapy in patients with premature $\mathrm{CAD}$, atherosclerosis and hypercholesterolemia.

\section{METHODS}

The study was carried out in 2019-2020 at Moscow City Clinical Hospital № 51. A total of 18 patients (4 women and 14 men) aged $53 \pm 6.7$ (35-65 years) eligible for intensive lipid-lowering therapy were enrolled. Inclusion criteria: clinical manifestations of CAD or peripheral artery disease in men aged under 55, and women aged under 60, or hyperlipidemia (LDL-C levels exceeding $4.9 \mathrm{mmol} / \mathrm{L}$ ). Exclusion criteria: no submitted informed consent. Fifteen patients had a history of acute myocardial infarction and coronary artery stent placement: two of them had multivessel disease and underwent coronary artery bypass grafting (CABG), others had peripheral artery disease or dyslipidemia. Among patients, 16 individuals (36.4\%) had hypertension, three individuals (6.8\%) had diabetes mellitus, 11 individuals $(25 \%)$ reported smoking at the time of enrollment, and four individuals $(9.1 \%)$ reported the history of smoking.

All patients were divided into three groups based on the fact of receiving lipid-lowering therapy at the time of enrollment (see Fig.). Dynamic changes in blood lipid levels were assessed prior to therapy and 4-8 weeks after starting treatment. The efficiency of initial statin therapy was analyzed in group $1(n=6)$. All patients in this group have had received no lipid-lowering medications prior to enrollment: at least three months prior to enrollment because of their own decision to discontinue treatment.

All patients in group $2(n=6)$ had a history of myocardial infarction in young age (the average age was $54.5 \pm 1.87$ years). At the time of enrollment, the patients received lipidlowering drugs prescribed by attending physicians outside the scope of the study, however, LDL-C target levels $(<1.4 \mathrm{mmol} / \mathrm{L})$ had not been achieved. Five people received $20-40 \mathrm{mg}$ doses of atorvastatin, and one person received 10 doses of rosuvastatin. Lipid-lowering therapy was adjusted, and the dose was escalated in all patients.

All patients receiving statins were analyzed separately (groups 1 and 2) ( $n=12$ ).

Group $3(n=7)$ included five men and two women (the average age was $52.29 \pm 9.1$ years) (one patient moved to group 3 after having completed the assessment in group 2 in accordance with the protocol). Five patients had a history of myocardial infarction. All of them received lipid-lowering drugs (atorvastatin 40-80 mg once daily, rosuvastatin $40 \mathrm{mg}$ once daily in combination with ezetimibe $10 \mathrm{mg}$ once daily or, in case of statin intolerance or contraindications, only ezetimibe $10 \mathrm{mg}$ once daily). Against the background of such therapy, all patients had $L D L-C$ levels exceeding $1.7 \mathrm{mmol} / \mathrm{L}$ at the time of enrollment. All patients were prescribed alirocumab 150 mg once daily for two weeks in accordance with the study protocol. Group 3 also included two patients with provisional or established diagnosis of familial hypercholesterolemia made in accordance with the Dutch Lipid Clinical Network Score. One of these patients could not take statins due to concomitant diseases of the hepatobiliary tree (chronic pancreatitis, hepatitis C) and elevated levels of transaminases. The second patient with stable CAD failed to achieve LDL-C target levels when receiving maximum tolerated statin doses.

Blood sampling for biochemical tests and mass spectrometry was performed on the day of enrollment (in the morning after 12-hour fasting) and after 4-8 weeks of therapy. Blood was collected from cubital vein into sterile Vacutainer tubes. Serum was obtained by blood centrifugation at $3000 \mathrm{rpm}$ for $15 \mathrm{~min}$. CLIMA MC-15 biochemistry analyzer (RAL; Spain) was used to determine the parameters of serum. The following reference values were used: total cholesterol $2.0-5.2 \mathrm{mmol} / \mathrm{L}$; LDL-C up to $3.3 \mathrm{mmol} / \mathrm{L}$; HDL-C $0.91-1.56 \mathrm{mmol} / \mathrm{L}$; serum triglycerides 0.50-1.70 mmol/L.

\section{Measuring plasma sphingolipid levels}

Sphingolipids were determined in blood plasma of the described above patients. During sample preparation blood was centrifuged at $3000 \mathrm{rpm}$ for $10 \mathrm{~min}, 1.5 \mathrm{ml}$ of supernatant were collected. Then $1.5 \mathrm{ml}$ of supernatant were centrifuged at $15,000 \mathrm{rpm}$ for $10 \mathrm{~min}$ in order to ensure complete sedimentation of cells. Lipids for mass spectrometry were extracted from blood plasma by the Bligh and Dyer method [9].

Mass spectrometric detection of various molecular species of sphingomyelins, ceramides and sphingoid bases (sphingosine and sphinganine) was performed with the TSQ Endura Triple Quadrupole Mass Spectrometer (Thermo Fisher Scientific; Germany) using the multiple reaction monitoring (MRM) technique, the pressure in the collision cell was 2.0 mTorr. Resolution of Q1 and Q3 was 1.2 Da. For ceramides, fragmentation of protonated and dehydrated parent molecules was performed at the energy of $20 \mathrm{eV}$ down to ion with $\mathrm{m} / \mathrm{z} 264.2 \mathrm{Da}$, the dwell time was $35 \mathrm{~ms}$. For sphingomyelins, fragmentation of protonated parent molecules was performed at the energy of $20 \mathrm{eV}$ down to ion with $\mathrm{m} / \mathrm{z} 184.1 \mathrm{Da}$, the dwell time was $35 \mathrm{~ms}$. For sphingosine and sphingosine deuterated standard (d7, Avanti; USA), fragmentation of protonated parent molecules was performed at the energy of $12.5 \mathrm{eV}$ down to ions with $\mathrm{m} / \mathrm{z}$ 259.3 and 252.3 Da respectively, the dwell time was $35 \mathrm{~ms}$. For 
sphinganine, fragmentation of protonated parent molecule was performed at the energy of $12.5 \mathrm{eV}$ down to ion with $\mathrm{m} / \mathrm{z} 266.3$ $\mathrm{Da}$, the dwell time was $35 \mathrm{~ms}$. The following parameters of the ionization source were used: heater temperature set to $300^{\circ} \mathrm{C}$, capillary temperature $340{ }^{\circ} \mathrm{C}$, sheath gas flow rate 45 arb (arbitrary units), auxiliary gas flow rate 13 arb, sweep gas flow rate 1 arb. Sphingosine $d 7$, sphinganine, sphingomyelin d18:1/16:0, sphingomyelin d18:1/18:0, ceramide d18:1/16:0, ceramide d18:1/18:1, ceramide d18:1/18:0, ceramide d18:1/24:1, ceramide d18:1/24:0 (Avanti; USA) were used as standards. Chromatographic separation was carried out using the Ultimate 3000 system (Thermo Fisher Scientific; Germany) and the Eclipse Plus C8 column $3.0 \times 150$ mm (Agilent; USA), the particle size was $3.5 \mu \mathrm{m}$. Temperature was set to $50^{\circ} \mathrm{C}$, and flow rate was set to $400 \mu \mathrm{L} / \mathrm{min}$. When determining sphingosine, ceramides and sphingomyelin, the following mobile phases were used: phase $A, 0.1 \%$ formic acid $(\mathrm{v} / \mathrm{v})$ in water, phase $\mathrm{B}$, $0.1 \%$ formic acid $(\mathrm{v} / \mathrm{v})$ in methanol $(55 \%$ of phase $\mathrm{B}$ at $0.7 \mathrm{~min}$, $100 \%$ of phase B at $7 \mathrm{~min}, 100 \%$ of phase B at $12 \mathrm{~min}, 55 \%$ of phase B from 13 to $17 \mathrm{~min}, 55 \%$ of phase B at $13 \mathrm{~min})$. When determining sphinganine, the following mobile phases were used: phase $A, 0.1 \%$ formic acid $(\mathrm{v} / \mathrm{v})$ in water, phase $\mathrm{B}, 50 \%$ methanol $+50 \%$ acetonitrile $+0.1 \%$ formic acid $(\mathrm{v} / \mathrm{v})(20 \%$ of phase $B$ at 1.5 min, $100 \%$ of phase $B$ at 2 min, $100 \%$ of phase $B$ at $7 \mathrm{~min}, 20 \%$ of phase $B$ at $7 \mathrm{~min}, 20$ of phase $B$ at $10 \mathrm{~min}$ ). The relative ceramide value was assessed using external calibration (method of standard addition). The Ceramide Porcine Brain 860052P ceramide mixture (Avanti; USA) containing $50 \%$ of $\mathrm{d} 18: 1 / 18: 050 \%$ and $20 \%$ of $\mathrm{d} 18: 1 / 24: 1$ was used as a standard. Calculations were performed based on the peak areas for MRM transitions $\mathrm{MH}+\cdot \rightarrow \mathrm{m} / \mathrm{z} 264.4 \mathrm{Da}$ and $(\mathrm{M}+\mathrm{H}-\mathrm{H} 2 \mathrm{O})+\cdot \rightarrow \mathrm{m} / \mathrm{z}$ 264.4 Da. The content of sphingosine d18:1 was determined by internal calibration (internal standard method, the standard was D-erythro-sphingosine d7, Sigma; USA) based on the sum of peak areas for MRM transitions $(\mathrm{m} / \mathrm{z} 300+\cdot \rightarrow \mathrm{m} / \mathrm{z} 252.3$ Da for non-deuterated and $\mathrm{m} / \mathrm{z}$ $307+\cdot \rightarrow \mathrm{m} / \mathrm{z} 259.3$ for deuterated sphingosine). The content of sphingosine d18:0 was defined using external calibration (the standard was DL-erythro-dihidrosphingosine, Sigma; USA) based on the peak areas for MRM transitions $\mathrm{m} / \mathrm{z}$ $302+\cdot \rightarrow \mathrm{m} / \mathrm{z} 266.3 \mathrm{Da}$.
CRS, described as a predictor for coronary artery disease mortality, was assessed using the previously reported scale involving the use of certain ceramide molecular species [8]. In order to access the ceramide risk, the following ratios were calculated: Cer16:0/Cer24:0, Cer18:0/Cer24:0, Cer24:1/ Cer24:0. The levels of Cer16:0, Cer18:0, Cer24:1, and the values of these ceramides' ratios with Cer24:0, were divided into quartiles. The patients falling within the third quartile were assigned score 1 for each of six parameters, and the patients falling within the fourth quartile were assigned score 2 . The total score was considered the ceramide risk score.

\section{Statistical analysis}

Statistical processing of the results was performed using the SPSS ver. 21.0 software package (IBM; USA). Quantitative variables were presented as mean and standard deviation $(\mathrm{M} \pm \mathrm{SD})$. All quantitative variables were tested for normality using the Shapiro-Wilk test. Distribution of all the quantitative variables was other than normal. The Wilcoxon signed-rank test was used to compare related samples. Significance of differences between two independent samples was determined using the Mann-Whitney test. Significance of correlations was assessed using the Spearman's rank correlation coefficient. The differences were considered significant when $p<0.05$ in all types of analysis.

\section{RESULTS}

Changes in cholesterol and sphingolipid levels were assessed by groups of patients at the time of lipid-lowering therapy initiation and adjustment (statins or alirocumab) (Table 1).

In group 1, a significant decrease in the levels of shortchain ceramides Cer 14:1 was observed ( $p=0.046)$. Longchain sphingomyelins SM 22:1, SM 22:0, SM 24:0 significantly decreased against the background of lipid-lowering therapy initiation in all patients of this group $(p=0.028)$.

In group 2, the levels of total cholesterol $(p=0.028), L D L-C$ ( $p=0.043$ ), long-chain sphingomyelins SM18:1, SM 24:1 and SM 26:1, ceramide Cer 16:1 $(p=0.028)$, and SM 22:1 $(p=0.046)$ significantly decreased against the background

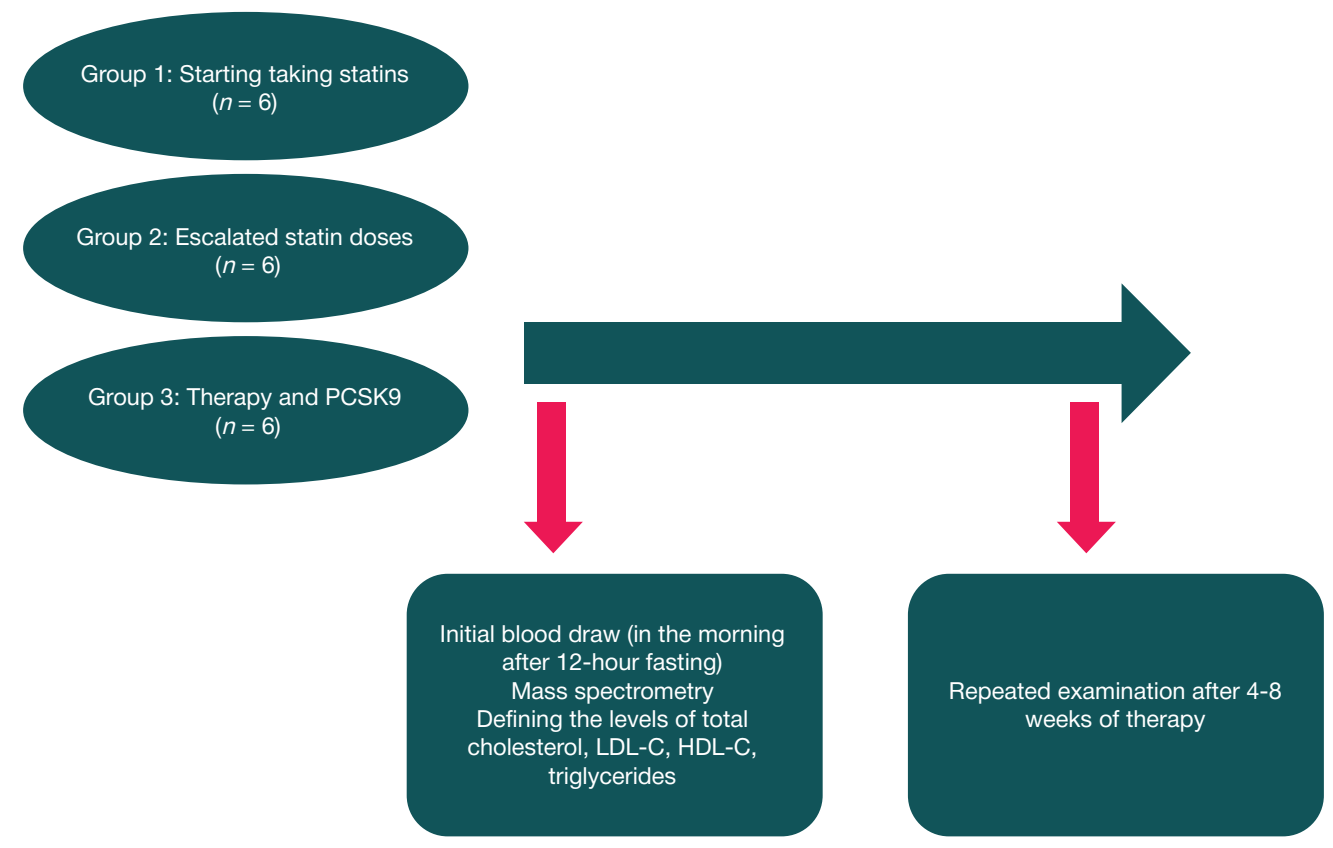

Figure. Study protocol 
Table 1. Dynamic changes in lipid levels in the studied groups

\begin{tabular}{|c|c|c|c|c|c|c|c|c|c|}
\hline \multirow{2}{*}{\multicolumn{2}{|c|}{ Indicators }} & \multicolumn{2}{|c|}{ Group $1(n=6)$} & \multicolumn{2}{|c|}{ Group $2(n=6)$} & \multicolumn{2}{|c|}{$\begin{array}{l}\text { All patients receiving statins } \\
\text { (groups } 1 \text { and 2) }(n=12)\end{array}$} & \multicolumn{2}{|c|}{ Group $3(n=7)$} \\
\hline & & $\mathrm{M} \pm \mathrm{SD}$ & $p$ & $\mathrm{M} \pm \mathrm{SD}$ & $p$ & $\mathrm{M} \pm \mathrm{SD}$ & $p$ & $\mathrm{M} \pm \mathrm{SD}$ & $p$ \\
\hline \multicolumn{10}{|c|}{ Standard lipid indicators ( $\mathrm{mmol} / \mathrm{L}$ ) } \\
\hline \multirow{2}{*}{ TC } & before & $7.42 \pm 2.39$ & \multirow{2}{*}{0.249} & $4.75 \pm 1.46$ & \multirow{2}{*}{$0.028^{*}$} & $6.09 \pm 2.35$ & \multirow{2}{*}{$0.028^{*}$} & $7.51 \pm 5.36$ & \multirow{2}{*}{0.063} \\
\hline & after & $5.83 \pm 1.38$ & & $4.31 \pm 1.36$ & & $5.07 \pm 1.53$ & & $4.79 \pm 1.49$ & \\
\hline \multirow{2}{*}{ LDL-C } & before & $4.98 \pm 2.45$ & \multirow{2}{*}{0.116} & $3.07 \pm 1.29$ & \multirow{2}{*}{$0.043^{*}$} & $4.03 \pm 2.12$ & \multirow{2}{*}{$0.021^{*}$} & $4.44 \pm 2.87$ & \multirow{2}{*}{$0.018^{\star}$} \\
\hline & after & $3.42 \pm 0.83$ & & $2.57 \pm 1.22$ & & $2.99 \pm 1.09$ & & $1.77 \pm 1.46$ & \\
\hline \multirow{2}{*}{ HDL-C } & before & $1.09 \pm 0.16$ & 0508 & $1.01 \pm 0.32$ & 0686 & $1.05 \pm 0.25$ & 0755 & $1.12 \pm 0.39$ & 0.176 \\
\hline & after & $1.18 \pm 0.33$ & $0.0<0$ & $0.98 \pm 0.23$ & 0.000 & $1.08 \pm 0.29$ & 0.150 & $1.51 \pm 1.08$ & 0.170 \\
\hline TG & before & $2.93 \pm 1.77$ & 0463 & $1.5 \pm 0.34$ & 0463 & $2.22 \pm 1.43$ & 0814 & $3.4 \pm 4.34$ & 0753 \\
\hline (n) & after & $2.69 \pm 2.20$ & 0.400 & $1.65 \pm 0.59$ & 0.400 & $2.17 \pm 1.63$ & 0.014 & $4.85 \pm 8.2$ & 0.100 \\
\hline & & & & CERAM & $(\mathrm{ng} / \mathrm{mL})$ & & & & \\
\hline & before & $0.96 \pm 0.16$ & & $0.71 \pm 0.09$ & & $0.83 \pm 0.18$ & & $0.39 \pm 0.42$ & \\
\hline & after & $0.7 \pm 0.11$ & (ס) & $0.76 \pm 0.09$ & 0.700 & $0.73 \pm 0.1$ & 0.100 & $0.49 \pm 0.4$ & 0.012 \\
\hline & before & $38.52 \pm 18.73$ & & $22.93 \pm 8.55$ & & $30.72 \pm 16.09$ & & $20.29 \pm 12.3$ & \\
\hline & after & $33.61 \pm 9.85$ & 0.400 & $23.81 \pm 6.93$ & 0.040 & $28.71 \pm 9.6$ & 1 & $22.3 \pm 5.42$ & 0.000 \\
\hline Cor $16 \cdot 1$ & before & $9.10 \pm 2.26$ & 0172 & $11.32 \pm 1.90$ & ०००००* & $10.21 \pm 2.31$ & 0027 & $7.17 \pm 3.35$ & 0610 \\
\hline Cer lo.1 & after & $13.26 \pm 6.34$ & 0.173 & $9.62 \pm 2.18$ & $0.0<0$ & $11.44 \pm 4.91$ & 0.931 & $7.09 \pm 2.96$ & 0.012 \\
\hline Cor $16 \cdot 0$ & before & $470.34 \pm 221.52$ & 06 & $222.07 \pm 23.74$ & 0753 & $346.2 \pm 198.42$ & 0583 & $370.49 \pm 353.84$ & 0612 \\
\hline 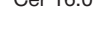 & after & $438.73 \pm 145.74$ & 0.0 & $222.27 \pm 55.34$ & 0.150 & $330.5 \pm 154.36$ & 0.000 & $338.64 \pm 240.77$ & 0.012 \\
\hline & before & $2.01 \pm 0.98$ & & $2.48 \pm 0.46$ & & $2.24 \pm 0.77$ & & $1.7 \pm 0.74$ & \\
\hline Cl & after & $2.37 \pm 0.49$ & 0.0 & $2.60 \pm 0.71$ & 0.0 & $2.48 \pm 0.6$ & 0.40 & $2.01 \pm 0.64$ & 0.170 \\
\hline Cor 100 & before & $45.10 \pm 31.72$ & 0402 & $40.67 \pm 7.68$ & $046 ?$ & $42.89 \pm 22.13$ & 0750 & $34.78 \pm 11.13$ & \\
\hline 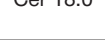 & after & $46.63 \pm 18.84$ & 0.400 & $38.31 \pm 7.83$ & 0.400 & $42.47 \pm 14.43$ & 0.154 & $33.36 \pm 9.92$ & 1 \\
\hline Cor 001 & before & $0.66 \pm 0.12$ & 0245 & $0.64 \pm 0.12$ & 0402 & $0.65 \pm 0.11$ & 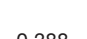 & $0.46 \pm 0.5$ & 0100 \\
\hline UEI 20.1 & after & $0.7 \pm 0.8$ & 0.040 & $0.71 \pm 0.16$ & 0.400 & $0.71 \pm 0.12$ & 0.000 & $0.59 \pm 0.2$ & 0.400 \\
\hline Cor 20 : & before & $71.84 \pm 36.27$ & 0017 & $81.88 \pm 23.78$ & 0017 & $76.86 \pm 29.71$ & 1 & $61.72 \pm 31.72$ & ( 0227 \\
\hline C 20.0 & after & $72.3 \pm 18.41$ & (1) & $78.59 \pm 17.31$ & (1) & $75.45 \pm 17.35$ & 1 & $81.18 \pm 50.40$ & $0 . \angle 01$ \\
\hline Cor 20.1 & before & $5.44 \pm 3.96$ & 0752 & $4.08 \pm 1.10$ & ०००००* & $4.76 \pm 2.86$ & 0180 & $3.47 \pm 2.34$ & ००००* \\
\hline Uet $2 \angle .1$ & after & $4.73 \pm 1.04$ & 0.100 & $5.62 \pm 0.81$ & $0.0<0$ & $5.18 \pm 1$ & 0.102 & $6.20 \pm 5.03$ & $0.0<0$ \\
\hline Cor 20.0 & before & $1948.4 \pm 1113.75$ & 0752 & $1254.62 \pm 181.13$ & 0215 & $1601.51 \pm 842.63$ & 0605 & $979.11 \pm 715.92$ & 0100 \\
\hline Uel $2<.0$ & after & $1821.5 \pm 765.99$ & 0.150 & $1358.95 \pm 299.06$ & 0.040 & $1590.22 \pm 604.74$ & 0.000 & $1336.47 \pm 743.56$ & 0.400 \\
\hline Cor $24 \cdot 1$ & before & $578.1 \pm 147.68$ & 0219 & $537.41 \pm 77.21$ & 0752 & $557.76 \pm 114.34$ & 0308 & $585.77 \pm 362.55$ & 0612 \\
\hline & after & $632.9 \pm 179.15$ & & $541.90 \pm 122.05$ & & $587.4 \pm 153.68$ & & $538.53 \pm 299.16$ & \\
\hline Corn 0 & before & $2083.59 \pm 1180.12$ & 0245 & $1451.87 \pm 143.86$ & 0017 & $1767.73 \pm 866.77$ & 0 & $1205.09 \pm 719.65$ & 0.176 \\
\hline $0.1<4.0$ & after & $1896.72 \pm 833.38$ & 0.040 & $1490.96 \pm 245.89$ & 0.911 & $1693.84 \pm 622.96$ & 0.48 & $1777.98 \pm 1078.81$ & $0.1 / 6$ \\
\hline Cor $26 \cdot 1$ & before & $12.50 \pm 8.24$ & 0116 & $13.10 \pm 8.14$ & 06 & $12.80 \pm 7.82$ & 0388 & $11.36 \pm 7.62$ & 0398 \\
\hline ver 20.1 & after & $16.35 \pm 8.14$ & 0.110 & $11.30 \pm 4.55$ & 0.0 & $13.83 \pm 6.82$ & 0.300 & $15.19 \pm 11.41$ & 0.390 \\
\hline Cor 20.0 & before & $49.67 \pm 7.68$ & 0017 & $45.48 \pm 3.33$ & 0017 & $47.57 \pm 6.05$ & 1 & $28.75 \pm 14.25$ & 201 \\
\hline Uel 20.0 & after & $50.28 \pm 5.59$ & 0.911 & $45.40 \pm 5.72$ & 0.911 & $47.84 \pm 5.96$ & 1 & $37.56 \pm 6.61$ & 0.01 \\
\hline Cer & before & $0.24 \pm 0.12$ & 0750 & $0.15 \pm 0.02$ & مe & $0.2 \pm 0.1$ & 0075 & $0.29 \pm 0.09$ & 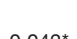 \\
\hline $16: 0 / 24: 0$ & after & $0.24 \pm 0.86$ & 0.153 & $0.15 \pm 0.03$ & 0.0 & $0.2 \pm 0.08$ & 0.075 & $0.19 \pm 0.04$ & 0.043 \\
\hline Cer & before & $0.028 \pm 0.027$ & 0315 & $0.03 \pm 0.01$ & 06 & $0.03 \pm 0.02$ & 0814 & $0.04 \pm 0.02$ & 0128 \\
\hline 18:0/24:0 & after & $0.03 \pm 0.01$ & 0.045 & $0.03 \pm 0.01$ & 0.0 & $0.03 \pm 0.02$ & 0.014 & $0.02 \pm 0.01$ & $0.1<0$ \\
\hline Cer & before & $0.33 \pm 0.16$ & (2000 & $0.37 \pm 0.03$ & 0017 & $0.35 \pm 0.11$ & 0207 & $0.58 \pm 0.28$ & ד \\
\hline $24: 1 / 24: 0$ & after & $0.37 \pm 0.17$ & 0.245 & $0.37 \pm 0.08$ & & $0.37 \pm 0.13$ & $0.04 t$ & $0.33 \pm 0.11$ & \\
\hline Ceramide & before & $4.83 \pm 3.71$ & 0336 & $2.67 \pm 2.07$ & 0503 & $3.75 \pm 3.08$ & 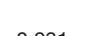 & $5.57 \pm 2.44$ & (2) \\
\hline risk score & after & $5.83 \pm 4.17$ & & $3.33 \pm 2.73$ & & $4.58 \pm 3.60$ & & $4.0 \pm 2.58$ & \\
\hline & & & & SPHINGON & INS (ng/ & & & & \\
\hline $\mathrm{CMM} 1 \mathrm{~A} 1$ & before & $1243.79 \pm 579.25$ & 0315 & $1076.05 \pm 456.93$ & 0345 & $1159.92 \pm 505.07$ & 0158 & $578.70 \pm 721.70$ & 0869 \\
\hline & after & $1107.35 \pm 539.53$ & & $969.59 \pm 329.81$ & & $1038.47 \pm 432.36$ & & $655.45 \pm 463.86$ & \\
\hline $\mathrm{CM} 11.0$ & before & $15560.92 \pm 7789.49$ & 0210 & $10218.99 \pm 4643.12$ & 0752 & $12889.95 \pm 6720.27$ & 0308 & $5379.57 \pm 6666.57$ & 0866 \\
\hline SIVI 14.0 & after & $13293.42 \pm 4925.96$ & 0.249 & $9803.30 \pm 3771.31$ & 0.150 & $11548.36 \pm 4562.52$ & 0.300 & $6516.82 \pm 4797.92$ & 0.000 \\
\hline & before & $27147.34 \pm 9245.24$ & & $14994.30 \pm 4552.79$ & & $21070.82 \pm 9410.35$ & & $9288.60 \pm 11795.93$ & \\
\hline SM 16:1 & after & $24289.12 \pm 8455.16$ & 0.345 & $14567.22 \pm 4838.38$ & 0.463 & $19428.17 \pm 8301.39$ & 0.209 & $12154.21 \pm 9026.99$ & 0.866 \\
\hline
\end{tabular}


Table 1 (continuation).

\begin{tabular}{|c|c|c|c|c|c|c|c|c|c|}
\hline \multirow{2}{*}{ SM 16:0 } & before & $68884.57 \pm 29300.16$ & \multirow{2}{*}{0.6} & $43109.35 \pm 5476.51$ & \multirow{2}{*}{0.249} & $55996.96 \pm 24187.79$ & \multirow{2}{*}{0.53} & $29457.23 \pm 26795.25$ & \multirow{2}{*}{0.612} \\
\hline & after & $58719.28 \pm 18464.01$ & & $41511.66 \pm 8959.72$ & & $50115.47 \pm 16498.73$ & & $42283.32 \pm 25753.31$ & \\
\hline \multirow{2}{*}{ SM 18:1 } & before & $4998.28 \pm 2970.69$ & \multirow{2}{*}{0.917} & $4138.09 \pm 957.21$ & \multirow{2}{*}{$0.028^{*}$} & $4568.18 \pm 2151.66$ & \multirow{2}{*}{0.182} & $3513.39 \pm 2645.09$ & \multirow{2}{*}{0.612} \\
\hline & after & $4254.22 \pm 2140.22$ & & $3786.43 \pm 1074.09$ & & $4020.32 \pm 1632.83$ & & $3483.78 \pm 1193.06$ & \\
\hline \multirow{2}{*}{ SM 18:0 } & before & $18597.76 \pm 10587.79$ & \multirow{2}{*}{0.345} & $14163.76 \pm 1608.14$ & \multirow{2}{*}{0.116} & $16380.76 \pm 7582.39$ & \multirow{2}{*}{0.071} & $8957.79 \pm 7701.24$ & \multirow{2}{*}{0.612} \\
\hline & after & $15006.64 \pm 5504.22$ & & $12963.34 \pm 2468.85$ & & $13984.99 \pm 4204.79$ & & $10214.96 \pm 5423.62$ & \\
\hline \multirow{2}{*}{ SM 20:1 } & before & $19571.11 \pm 9063.27$ & \multirow{2}{*}{0.463} & $34801.84 \pm 6713.68$ & \multirow{2}{*}{0.249} & $27186.47 \pm 11004.16$ & \multirow{2}{*}{0.182} & $19003.09 \pm 7981.50$ & \multirow{2}{*}{0.499} \\
\hline & after & $15399.44 \pm 4090.53$ & & $33308.30 \pm 7232.94$ & & $24353.87 \pm 10902.14$ & & $21255.95 \pm 11508.78$ & \\
\hline \multirow{2}{*}{ SM 20:0 } & before & $122191.42 \pm 61041.05$ & \multirow{2}{*}{0.463} & $107398.59 \pm 12787.35$ & \multirow{2}{*}{0.463} & $114795.00 \pm 42750.99$ & \multirow{2}{*}{0.239} & $83678.97 \pm 38585.26$ & \multirow{2}{*}{0.31} \\
\hline & after & $95985.48 \pm 23943.68$ & & $104156.92 \pm 15085.34$ & & $100071.20 \pm 19550.99$ & & $108695.09 \pm 50687.07$ & \\
\hline \multirow{2}{*}{ SM 22:1 } & before & $32751.55 \pm 7675.39$ & \multirow{2}{*}{$0.028^{*}$} & $37536.42 \pm 11536.91$ & \multirow{2}{*}{$0.046^{*}$} & $35143.98 \pm 9670.69$ & \multirow{2}{*}{$0.004^{*}$} & $14233.44 \pm 15712.63$ & \multirow{2}{*}{0.866} \\
\hline & after & $27480.53 \pm 7931.12$ & & $33221.35 \pm 10422.30$ & & $30350.94 \pm 9324.97$ & & $18164.23 \pm 11614.33$ & \\
\hline \multirow{2}{*}{ SM 22:0 } & before & $49975.11 \pm 7453.47$ & $0 \cap 28^{*}$ & $50443.83 \pm 11076.52$ & 0753 & $50209.47 \pm 9004.42$ & 0.071 & $17147.95 \pm 18892.94$ & \\
\hline & after & $39664.55 \pm 10888.20$ & O.0.0 & $49134.09 \pm 16770.24$ & 0.100 & $44399.32 \pm 14358.99$ & ( & $25262.43 \pm 16913.88$ & $r$ \\
\hline & before & $86437.81 \pm 20948.28$ & & $87494.43 \pm 21994.99$ & & $86966.12 \pm 20485.92$ & & $31116.71 \pm 31595.14$ & \\
\hline Sivi $<4.1$ & after & $80493.43 \pm 14190.94$ & 0.170 & $76736.87 \pm 22166.55$ & $0.0<0$ & $78615.15 \pm 17853.01$ & 0.012 & $43885.51 \pm 28272.08$ & 1 \\
\hline SM 24.0 & before & $44680.38 \pm 11292.01$ & $0028^{*}$ & $41334.09 \pm 7361.87$ & 0.463 & $43007.24 \pm 9254.61$ & 0028 * & $11042.64 \pm 12530.05$ & 0735 \\
\hline 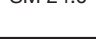 & after & $33338.06 \pm 6060.00$ & 0.020 & $38593.57 \pm 12040.46$ & 0.700 & $35965.82 \pm 9493.26$ & 年 & $15850.33 \pm 10223.83$ & 0.100 \\
\hline & before & $992.02 \pm 541.84$ & & $944.03 \pm 417.87$ & & $968.02 \pm 462.01$ & & $248.38 \pm 269.04$ & \\
\hline Civi & after & $769.60 \pm 301.79$ & 0.170 & $752.44 \pm 269.19$ & $0.0<0$ & $761.02 \pm 272.79$ & 0.070 & $398.84 \pm 285.35$ & 0.700 \\
\hline & & & & SPHINGOID & SES (ng & & & & \\
\hline SPN & before & $5.26 \pm 6.71$ & 0345 & $3.18 \pm 1.59$ & 075 & $4.22 \pm 4.77$ & 04 & $2.30 \pm 1.21$ & 1735 \\
\hline 年 & after & $3.20 \pm 0.92$ & (.0. & $3.28 \pm 1.14$ & . & $3.24 \pm 0.99$ & . & $2.47 \pm 1.50$ & 0.00 \\
\hline$S B$ & before & $13.32 \pm 17.58$ & 0 & $9.39 \pm 4.25$ & 0 & $11.35 \pm 12.36$ & 0388 & $7.00 \pm 2.98$ & 237 \\
\hline 年 & after & $10.85 \pm 5.28$ & . & $9.85 \pm 4.61$ & . & $10.35 \pm 4.75$ & 0.000 & $8.27 \pm 4.78$ & 年 \\
\hline S1 & before & $17.28 \pm 5.13$ & 0753 & $19.32 \pm 2.88$ & 011 & $18.30 \pm 4.11$ & 0.433 & $14.57 \pm 2.75$ & 0 31 \\
\hline - & after & $16.84 \pm 2.01$ & (1.00 & $17.54 \pm 1.50$ & (5) & $17.19 \pm 1.73$ & . & $16.18 \pm 3.61$ & 0.0 \\
\hline
\end{tabular}

Note: SPN — sphingosine, SPH — sphingonine, S1P — sphingosine-1-phosphate.

of the escalated statin doses. Cer 22:1 levels significantly increased $(p=0.028)$.

After the lipid-lowering therapy adjustment, the levels of cholesterol decreased by $9.5 \%$ ( $\triangle$ cholesterol $=-0.45 \pm 0.40$ ), and the levels of LDL-C decreased by $16.2 \%(\triangle \mathrm{LDL}-\mathrm{C}=-0.50$ $\pm 0.44)$ in all patients. LDL-C target levels were achieved in only one patient, in other patients, advice was provided on lifestyle correction, the lipid-lowering drug dose was escalated, or the second drug was prescribed (ezetimibe $10 \mathrm{mg}$ once daily). One female patient maintained LDL-C levels $\geq 2.6 \mathrm{mmol} / \mathrm{L}$ against the background of dual lipid-lowering therapy (maximum tolerated doses of statins: atorvastatin $80 \mathrm{mg}$, ezetimibe $10 \mathrm{mg}$ ). She was prescribed alirocumab.

Analysis of data obtained in all patients receiving statins revealed significantly decreased levels of cholesterol $(p=0.028)$, LDL-C $(p=0.021)$, and long-chain sphingomyelins SM 24:1 $(p=0.012), \operatorname{SM} 24: 0(p=0.028), \operatorname{SM} 26: 1(p=0.010)$, SM 22:1 $(p=0.004)$.

In group 3, the levels of total cholesterol decreased by $36.2 \%(\triangle T C=-2.72 \pm 4.48)$, and the levels of LDL-C $(p=0.018)$ decreased by $60.1 \%$ compared to baseline $(\triangle \mathrm{LDL}-\mathrm{C}=-2.67 \pm$ 3.12) against the background of alirocumab therapy.

Of all the defined ceramide ratios (Cer 16:0/24:0, 18:0/24:0, 24:1/24:0), only the Cer 16:0/24:0 ratio significantly decreased in the group receiving alirocumab $(p=0.043)$. No significant changes in ceramide risk score were observed in any of the groups.

Comparison of dynamic changes in sphingolipid levels was performed in patients, who had started therapy for the first time, and against the background of escalated statin doses (groups 1 and 2), as well as in each group of patients receiving alirocumab (group 3) (Table 2).

When comparing dynamic changes in sphingolipid levels in each group, it was observed that in group 1 the level of ceramide Cer 16:1 increased slightly $(\triangle$ Cer 16:1 $=4.1 \pm 7.17)$, and in group 2 it decreased ( $\triangle$ Cer 16:1 $=-1.70 \pm 1.32$ ). The differences were considered significant $(p=0.015)$.

Against the background of alirocumab therapy, the increase in the levels of Cer 14:1 $(\triangle$ Cer 14:1 $=0.10 \pm 0.35)$ was observed $(p=0.022)$, however, the levels of this ceramide decreased $(\triangle$ Cer 14:1 $=-0.26 \pm 0.259$ ) in patients, who had started taking statins. When comparing dynamic changes in groups 1 and 3 , the significant differences were observed in the levels of SM 22:0 ( $\triangle$ SM 22:0 $=-10310.6$ vs 8114.48) $(p=0.035)$ and SM 24:0 ( $\triangle \mathrm{SM} 24: 0=-11342.31$ vs 4807.69) $(p=0.022)$. In patients receiving statins and patients receiving alirocumab the dynamic changes of SM 22:1 ( $\triangle \mathrm{SM} 22: 1=$ -4793.04 vs 3930.79) $(p=0.045)$ and SM 26:1 ( $\triangle \mathrm{SM} 26: 1=$ -207.00 vs 150.46) ( $p=0.005)$ were different in groups 2 and 3 ( $\triangle \mathrm{SM} 26: 1=-191.58$ vs 150.46).

When conducting correlation analysis in group 1, a significant strong positive correlation was observed between the dynamic changes of Cer 14:1 and the changes in the levels of LDL-C ( $\rho=0.829 ; p=0.042)$; there was a significant strong negative correlation with baseline LDL-C level $(\rho=-0.829$; $p=0.042$ ) (Table 3). In the same group, a positive correlation was observed between the dynamic changes of long-chain sphingomyelins, and the changes in the levels of $\mathrm{LDL}-\triangle$ SM 24:1 with $\Delta$ LDL $(\rho=0.943 ; p=0.005), \triangle$ SM 26:1 with $\triangle \operatorname{LDL}(\rho=0.829 ; p=0.042)$, together with negative correlation 
Table 2. Dynamic changes of the lipidome in the studied groups

\begin{tabular}{|c|c|c|c|c|c|c|c|c|}
\hline & $\begin{array}{c}\text { Group 1 } \\
(n=6)\end{array}$ & $\begin{array}{c}\text { Group } 2 \\
(n=6)\end{array}$ & $\begin{array}{l}\text { All patients receiving statins } \\
\text { (groups } 1 \text { and } 2 \text { ) }\end{array}$ & $\begin{array}{c}\text { Group } 3 \\
(n=7)\end{array}$ & \multicolumn{4}{|c|}{$p$} \\
\hline & $\mathrm{M} \pm \mathrm{SD}$ & $\mathrm{M} \pm \mathrm{SD}$ & $\mathrm{M} \pm \mathrm{SD}$ & $\mathrm{M} \pm \mathrm{SD}$ & 1 vs 2 & 1 vs 3 & 2 vs 3 & $1+2$ vs 3 \\
\hline $\begin{array}{c}\Delta \text { Total cholesterol, } \\
\mathrm{mmol} / \mathrm{L}\end{array}$ & $-1.59 \pm 2.26$ & $-0.45 \pm 0.40$ & $-1.02 \pm 1.66$ & $-2.72 \pm 4.48$ & 1 & 1 & 0.366 & 0.592 \\
\hline$\Delta \mathrm{LDL}-\mathrm{C}, \mathrm{mmol} / \mathrm{L}$ & $-1.57 \pm 2.05$ & $-0.50 \pm 0.44$ & $-1.03 \pm 1.52$ & $-2.67 \pm 3.12$ & 0.589 & 0.445 & 0.101 & 0.142 \\
\hline \multicolumn{9}{|c|}{ CERAMIDES (ng/mL) } \\
\hline$\Delta$ Cer $14: 1$ & $-0.26 \pm 0.259$ & $0.05 \pm 0.10$ & $-0.10 \pm 0.25$ & $0.10 \pm 0.35$ & 0.065 & $0.022^{*}$ & 0.731 & 0.299 \\
\hline$\Delta$ Cer 14:0 & $-4.9 \pm 16.8$ & $0.87 \pm 6.07$ & $-2.01 \pm 12.41$ & $2.01 \pm 14.44$ & 0.699 & 0.445 & 0.534 & 0.967 \\
\hline$\Delta$ Cer $16: 1$ & $4.1 \pm 7.17$ & $-1.70 \pm 1.32$ & $1.23 \pm 5.79$ & $-0.08 \pm 5.64$ & $0.015^{\star}$ & 0.181 & 0.945 & 0.482 \\
\hline$\Delta$ Cer $16: 0$ & $-31.61 \pm 128.6$ & $0.20 \pm 39.22$ & $-15.70 \pm 92.17$ & $-31.85 \pm 186.90$ & 0.485 & 0.731 & 0.295 & 0.384 \\
\hline$\Delta$ Cer 18:1 & $0.36 \pm 1.44$ & $0.12 \pm 1.10$ & $0.24 \pm 1.23$ & $0.31 \pm 0.87$ & 0.699 & 1 & 0.731 & 0.837 \\
\hline$\Delta$ Cer 18:0 & $1.53 \pm 22.4$ & $-2.36 \pm 8.61$ & $-0.41 \pm 16.33$ & $-1.42 \pm 14.20$ & 0.24 & 0.534 & 0.945 & 0.773 \\
\hline$\triangle$ Cer 20:1 & $0.04 \pm 0.96$ & $0.07 \pm 0.22$ & $0.05 \pm 0.16$ & $0.13 \pm 0.49$ & 0.937 & 0.534 & 0.836 & 0.592 \\
\hline$\Delta$ Cer 20:0 & $0.46 \pm 31.2$ & $-3.30 \pm 23.91$ & $-1.42 \pm 26.57$ & $19.45 \pm 33.98$ & 1 & 0.366 & 0.366 & 0.261 \\
\hline$\Delta$ Cer 22:1 & $-0.71 \pm 3.12$ & $1.55 \pm 0.86$ & $0.42 \pm 2.48$ & $2.73 \pm 3.13$ & 0.24 & 0.073 & 0.731 & 0.196 \\
\hline$\Delta$ Cer 22:0 & $-126.9 \pm 455.6$ & $104.33 \pm 253.10$ & $-11.29 \pm 371.56$ & $357.35 \pm 684.10$ & 0.699 & 0.366 & 0.628 & 0.837 \\
\hline$\Delta$ Cer 24:1 & $54.8 \pm 80.02$ & $4.48 \pm 76.78$ & $29.64 \pm 79.25$ & $-47.24 \pm 276.47$ & 0.24 & 0.366 & 0.836 & 0.482 \\
\hline$\Delta$ Cer 24:0 & $-186.9 \pm 377$ & $39.09 \pm 303.56$ & $-73.89 \pm 347.01$ & $572.89 \pm 946.70$ & 0.394 & 0.366 & 0.731 & 0.432 \\
\hline$\Delta$ Cer 26:1 & $3.85 \pm 5.3$ & $-1.80 \pm 8.47$ & $1.03 \pm 7.35$ & $3.83 \pm 8.54$ & 0.31 & 0.731 & 0.445 & 0.837 \\
\hline$\Delta$ Cer 26:0 & $0.60 \pm 9.61$ & $-0.09 \pm 3.01$ & $0.26 \pm 6.80$ & $8.82 \pm 16.90$ & 0.818 & 0.534 & 0.731 & 0.536 \\
\hline \multicolumn{9}{|c|}{ SPHINGOMYELINS, (ng/mL) } \\
\hline$\triangle \mathrm{SM} 14: 1$ & $-136.44 \pm 264.64$ & $-106.46 \pm 222.84$ & $-121.45 \pm 233.78$ & $76.75 \pm 597.50$ & 1 & 0.945 & 0.628 & 0.711 \\
\hline$\triangle \mathrm{SM} 14: 0$ & $-2267.50 \pm 4319.4$ & $-415.69 \pm 2599.93$ & $-1341.60 \pm 3533.89$ & $1137.25 \pm 7185.34$ & 0.394 & 0.534 & 0.731 & 0.536 \\
\hline$\triangle \mathrm{SM} 16: 1$ & $-2858.22 \pm 5090.2$ & $-427.07 \pm 2668.56$ & $-1642.65 \pm 4077.53$ & $2865.61 \pm 12002.14$ & 0.589 & 1 & 0.945 & 0.967 \\
\hline$\triangle \mathrm{SM} 16: 0$ & $-10165.3 \pm 42892.64$ & $-1597.70 \pm 4740.43$ & $-5881.49 \pm 29436.31$ & $12826.09 \pm 39663.43$ & 1 & 0.445 & 0.945 & 0.592 \\
\hline$\triangle \mathrm{SM} 18: 1$ & $-744.05 \pm 3065.24$ & $-351.66 \pm 255.92$ & $-547.86 \pm 2083.88$ & $-29.60 \pm 2322.63$ & 0.394 & 0.366 & 0.234 & 0.196 \\
\hline$\triangle \mathrm{SM} 18: 0$ & $-3591.13 \pm 9189.7$ & $-1200.41 \pm 1637.43$ & $-2395.77 \pm 6415.91$ & $1257.17 \pm 7871.83$ & 0.699 & 0.366 & 0.445 & 0.299 \\
\hline$\triangle \mathrm{SM} 20: 1$ & $-4171.7 \pm 10715.9$ & $-1493.53 \pm 3174.60$ & $-2832.60 \pm 7663.77$ & $2252.86 \pm 10836.10$ & 0.699 & 0.295 & 0.366 & 0.227 \\
\hline$\triangle \mathrm{SM} 20: 0$ & $-26205.9 \pm 68374.9$ & $-3241.66 \pm 9337.83$ & $-14723.80 \pm 48047.04$ & $25016.12 \pm 54734.68$ & 0.699 & 0.234 & 0.234 & 0.142 \\
\hline$\triangle \mathrm{SM} 22: 1$ & $-5271.02 \pm 2725.40$ & $-4315.07 \pm 3580.24$ & $-4793.04 \pm 3074.40$ & $3930.79 \pm 13493.31$ & 0.485 & 0.101 & 0.101 & $0.045^{*}$ \\
\hline$\triangle \mathrm{SM} 22: 0$ & $-10310.6 \pm 7398.5$ & $-1309.75 \pm 15485.82$ & $-5810.15 \pm 12489.23$ & $8114.48 \pm 20690.12$ & 0.24 & $0.035^{*}$ & 0.534 & 0.1 \\
\hline$\triangle \mathrm{SM} 24: 1$ & $-5944.38 \pm 9369.28$ & $-10757.56 \pm 7584.82$ & $-8350.97 \pm 8507.03$ & $12768.79 \pm 34445.18$ & 0.485 & 0.445 & 0.138 & 0.167 \\
\hline$\triangle \mathrm{SM} 24: 0$ & $-11342.31 \pm 7409.98$ & $-2740.53 \pm 7497.64$ & $-7041.42 \pm 8407.70$ & $4807.69 \pm 13661.89$ & 0.132 & $0.022^{*}$ & 0.445 & 0.068 \\
\hline$\triangle \mathrm{SM} 26: 1$ & $-222.42 \pm 267.17$ & $-191.58 \pm 204.63$ & $-207.00 \pm 227.46$ & $150.46 \pm 270.72$ & 1 & 0.073 & $0.005^{*}$ & $0.005^{*}$ \\
\hline
\end{tabular}

with baseline LDL-C level - with $\triangle$ SM 24:1 $(\rho=-0.943$; $p=0.005)$, with $\triangle \mathrm{SM} 26: 1$ ( $\rho=-0.829 ; p=0.042)$. In group 2 , only changes in SM 18:0 positively correlated with baseline LDL-C level $(\rho=0.812 ; p=0.05)$.

In group 1, there was a significant strong correlation between the dynamic changes in $\triangle \mathrm{SM} 26: 1$ and the baseline HDL-C level ( $\rho=-0.829 ; p=0.042)$; no significant correlations were observed in patients of group 2. Significant correlations with baseline HDL-C level in all patients receiving statins were observed for $\triangle$ SM 14:1 ( $\rho=-0.602 ; p=0.038), \Delta$ SM 14:0 $(\rho=-0.676 ; p=0.016), \triangle \operatorname{SM~16:1}(\rho=-0.630 ; p=0.028)$, $\triangle$ SM 24:0 $(\rho=-0.581 ; p=0.047, \triangle$ SM 26:1 $\rho=-0.595$; $p=0.041)$. In group 3 , there was a significant strong negative correlation between changes in SM 20:1 ( $\rho=-0.929 ; p=0.003)$ and SM 20:0 ( $\rho=-0.929 ; p=0.003)$ and baseline HDL-C level.

\section{DISCUSSION}

Currently, the major classes of lipid-lowering drugs are inhibitors of HMG-CoA reductase (statins) inhibiting hepatic cholesterol biosynthesis, cholesterol absorption inhibitors (ezetimibe), and proprotein convertase subtilisin/kexin type 9 (PCSK9) inhibitors acting through inhibiting of PCSK9, binding to LDL receptor, and the increase in the number of such receptors on the surface of hepatocytes. When applying lipid-lowering therapy, the main clinical reference is the decrease in the LDL-C levels to target levels [2]. The differences in the mechanism of action may also contribute to differences in effect on the levels of other lipid fractions, such as sphingomyelins and ceramides. It is known, that ceramide levels may be considered as an additional risk factor for complications of atherosclerosis (myocardial infarction and stroke). This has been shown in the cohort study Rochester Epidemiology Project (REP) when observing 1131 healthy individuals aged over 45 . The ratios Cer 16:0/Cer 24:0, Cer 18:0/Cer 24:0, Cer 24:1/Cer 24:0, and the ceramide risk score were the main predictors of adverse outcomes [10]. The same ceramides and their ratios were found to be independent predictors of cardiovascular death in the cohort of 1704 patients with CAD [11]. In the course of our study, we also assessed the dynamic changes of these ratios and the ceramide risk score against the background of lipid-lowering therapy.

Elevated sphingomyelin levels can be also considered as an independent predictor of adverse outcomes. This has been shown in analysis of cohorts of patients with familial lipid 
Table 3. Correlation analysis of dynamic changes with baseline levels of LDL-C, HDL-C, and dynamic changes of LDL-C

\begin{tabular}{|c|c|c|c|}
\hline & Baseline LDL-C & $\triangle$ LDL-C & Baseline HDL-C \\
\hline \multicolumn{4}{|c|}{ Group $1(n=6)$} \\
\hline$\Delta$ Cer $14: 1$ & $\rho=-0.829 ; p=0.042$ & $\rho=0.829 ; p=0.042$ & $\rho=-0.6 ; p=0.208$ \\
\hline$\triangle \mathrm{SM} 24: 1$ & $\rho=-0.943 ; p=0.005$ & $\rho=0.943 ; p=0.005$ & $\rho=-0.657 ; p=0.156$ \\
\hline$\triangle \mathrm{SM} 26: 1$ & $\rho=-0.829 ; p=0.042$ & $\rho=0.829 ; p=0.042$ & $\rho=-0.829 ; p=0.042$ \\
\hline \multicolumn{4}{|c|}{ Group $2(n=6)$} \\
\hline$\triangle \mathrm{SM} 18: 0$ & $\rho=0.812 ; p=0.05$ & $\rho=-0.200 ; p=0.704$ & $\rho=0.029 ; p=0.957$ \\
\hline \multicolumn{4}{|c|}{ All patients receiving statins (groups 1 and 2) $(n=12)$} \\
\hline$\triangle \mathrm{SM} \mathrm{14:1}$ & $\rho=-0.329 p=0.296$ & $\rho=0.606 ; p=0.037$ & $\rho=-0.602 ; p=0.038$ \\
\hline$\triangle \mathrm{SM} \mathrm{14:0}$ & $\rho=-0.417 ; p=0.178$ & $\rho=0.504 p=0.094$ & $\rho=-0.676 ; p=0.016$ \\
\hline$\triangle \mathrm{SM} \mathrm{16:1}$ & $\rho=-0.109 ; p=0.737$ & $\rho=0.245 ; p=0.442$ & $\rho=-0.630 ; p=0.028$ \\
\hline$\triangle \mathrm{SM} 24: 0$ & $\rho=-0.235 ; p=0.463$ & $\rho=0.343 ; p=0.275$ & $\rho=-0.581 ; p=0.047$ \\
\hline$\triangle \mathrm{SM} 26: 1$ & $\rho=-0.452 ; p=0.140$ & $\rho=0.480 ; p=0.114$ & $\rho=-0.595 ; p=0.041$ \\
\hline \multicolumn{4}{|c|}{ Group $3(n=7)$} \\
\hline$\triangle \mathrm{SM} 20: 1$ & $\rho=-0.143 ; p=0.760$ & $\rho=-0.036 ; p=0.939$ & $\rho=-0.929 ; p=0.003$ \\
\hline$\triangle \mathrm{SM} 20: 0$ & $\rho=-0.179 ; p=0.702$ & $\rho=0.107 ; p=0.819$ & $\rho=-0.929 ; p=0.003$ \\
\hline
\end{tabular}

disorders using the methods of big data analysis and machine learning [12]. Prognostic value of certain sphingomyelins has been also shown for cerebrovascular disease [13].

There is little literature data on the effect of therapy with statins, PCSK9 inhibitors, and ezetimibe on the sphingolipid levels. The randomized trial showed that the combination of atorvastatin and fibrates ensured reduced levels of acylglycerols and the majority of ceramides, as well as the elevated levels of sphingomyelins. Atorvastatin dose escalation modestly decreased the levels of lysophosphatidylcholine [14] The randomized trial aimed to compare the effect of $80 \mathrm{mg}$ simvastatin and $10 \mathrm{mg}$ simvastatin/ezetimibe combination showed the differences in the effects of these regimens on the levels of lipid and sphingolipid fractions. High-dose statin therapy resulted in more prominent changes in sphingolipid levels. Meanwhile, the dynamic changes in the cholesterol and LDL-C levels were comparable. The high-dose statin reduced the levels of sphingomyelins and ceramides much more; it also affected the levels of phosphotidylcholine, which could partially explain the pleiotropic effect of statins [15].

In the course of randomized trial involving the use of different rosuvastatin doses (10 and $40 \mathrm{mg}$ ) in patients with metablic syndrome, both low and high statin doses significantly decreased the levels of ceramides and sphingolipids. The significant dose-dependent differences were shown for dynamic changes of phosphatidylcholine, lysophosphatidylcholine, alkylphosphatidylcholine, alkenylphosphatidylcholine (plasmalogen), and phosphatidylinositol. The dose-dependent effect was also shown for sphingolipids after being normalized to phosphatidylcholine level [16].

During treatment with rosuvastatin, the dynamic changes in blood ceramide levels negatively correlated with the dynamic changes in the levels of ApoB100 very low-density lipoproteins (VLDL). No association between the dynamic changes in the levels of ceramides and sphingomyelins, and the dynamic changes in the levels of total cholesterol, LDL-C, triglycerides, and ApoA1 LDL was observed. The discovered association between the dynamic changes in ApoB VLDL and the dynamic changes in ceramides against the background of rosuvastatin therapy demonstrates the potential features of the mechanism of action for this statin [17].

In our study, the initial treatment with statins resulted in significantly decreased levels of Cer 14:1 and reduced levels of sphingomyelins SM 22:1, SM 22:0, and SM 24:0. Reduced levels of Cer 14:1 and long-chain sphingomyelins correlated with baseline levels of LDL-C and dynamic changes in LDL. With the lipid-lowering treatment intensification (escalation of statin dose and addition of ezetimibe in some patients), the overall dynamic changes in sphingomyelins were more prominent, which correlated with the results of the studies cited. No significant impact on the levels of ceramides and ceramide risk was observed.

Association between PCSK9 level and blood ceramide and sphingolopid levels was investigated in the course of the study, involoving 31 patients with liver diseases of different etiology. In patients with high PCSK9 level, LDL-C levels were higher than expected. However, the levels of total cholesterol, sphingolopids, and ceramides showed no correlation with PCSK9 level. After the patients were divided into groups with high and low PCSK9 levels, it turned out that the patients with plasma PCSK9 levels above the median had significantly lower levels of certain sphingolipids and ceramides: CE 16:0, CE 20:5, CE 20:4, CE 22:6, CE 22:4, SM 18:0, SM 20:1, SM 24:2, $\mathrm{SM} 24: 1$. To date, the mechanism, underlying such association, is unclear [18].

The EQUATOR trial, involving the use of monoclonal antibody against PCSK9 RG7652, showed the predominant decline in the levels of ceramides CE 24:1, CE 24:0, and CE 26:0, contained mainly in LDL [19]. The other study, involving PCSK9 inhibitor prescription against the background of treatment at the maximum tolerated statin dose, also showed both reduced ceramide levels and reduced ceramide risk score, the integral parameter, which correlated with the risk of adverse outcome in patients with dyslipidemia [20]. Earlier, the Ludwigshafen Risk and Cardiovascular Health (LURIC) trial showed that homozygous state for the rare mutant allele R46L (PCSK9), characterized by low enzyme activity, was associated with $30 \%$ decrease in the ceramide risk score compared to other allelic variants [21]. This trial also demonstrated $13 \%$ decrease in the ceramide risk score against the background of simvastatin therapy, and lack of ceramide dynamic changes against the background of taking ezetimibe.

During our study, alirocumab added to therapy resulted in significantly decreased LDL-C, however, no significant changes in the levels of sphingomyelins and ceramides were observed. Only significant increase in Cer 22:1 and decreased ratio Cer 
16:0/24:0, being the marker of ceramide risk, were observed. In the course of our study, alirocumab was added to therapy in patients, who had previously received maximum tolerated statin doses in accordance with indications registered for appropriate drug [2, 22]. However, the baseline sphingolipid levels were relatively low; these were significantly lower compared to groups 1 and 2 against the background of four-week statin therapy. Against the background of low baseline sphingomyelin levels, PCSK9 inhibitor had no significant effect of the levels of sphingomyelins and ceramides. It was probably due to the drug mechanism of action, different from the statin mechanism of action.

This study had the following limitations: the sample of patients was small, the study was conducted with the use of background therapy with various statins.

\section{CONCLUSION}

Sphingomyelin levels decrease against the background of statin therapy; when applying starting doses of statins, these correlate with reduced levels of LDL-C. When escalating lipidlowering therapy, the correlation between dynamic changes in sphingomyelins and LDL becomes less prominent. Treatment with PCSK9 inhibitors results in significantly reduced LDL-C levels, however, it does not affect the levels of sphingomyelins. No significant dynamic changes in ceramides and ceramide risk are observed against the background of statin therapy. However, PCSK9 inhibitor added to therapy reduces the Cer 16:0/24:0 ratio, which can be considered a marker of decreased cardiovascular risk.

\section{References}

1. Bossard M, Latifi Y, Fabbri M, Kurmann R, Brinkert M, Wolfrum M, et al. Increasing Mortality From Premature Coronary Artery Disease in Women in the Rural United States. J Am Heart Assoc. 2020; 9 (9): e015334. DOI: 10.1161/JAHA.119.015334.

2. Mach F, Baigent C, Catapano AL, Koskinas KC, Casula M, Badimon L, et al; ESC Scientific Document Group. 2019 ESC/ EAS Guidelines for the management of dyslipidaemias: lipid modification to reduce cardiovascular risk. Eur Heart J. 2020; 41 (1): 111-88. DOI: 10.1093/eurheartj/ehz455.

3. Ference BA, Ginsberg HN, Graham I, Ray KK, Packard CJ, Bruckert E, et al. Low-density lipoproteins cause atherosclerotic cardiovascular disease. 1. Evidence from genetic, epidemiologic, and clinical studies. A consensus statement from the European Atherosclerosis Society Consensus Panel. Eur Heart J. 2017; 38 (32): 2459-72. DOI: 10.1093/eurheartj/ehx144.

4. Khera AV, Won HH, Peloso GM, Lawson KS, Bartz TM, Deng X, et al. Diagnostic Yield and Clinical Utility of Sequencing Familial Hypercholesterolemia Genes in Patients With Severe Hypercholesterolemia. J Am Coll Cardiol. 2016; 67 (22): 2578-89. DOI: 10.1016/j.jacc.2016.03.520.

5. Barenholz Y. Sphingomyelin and cholesterol: from membrane biophysics and rafts to potential medical applications. Subcell Biochem. 2004; 37: 167-215. DOl: 10.1007/978-1-4757-5806-1_5.

6. Manicke NE, Nefliu M, Wu C, Woods JW, Reiser V, Hendrickson RC, et al. Imaging of lipids in atheroma by desorption electrospray ionization mass spectrometry. Anal Chem. 2009; 81 (21): 8702-7. DOI: 10.1021/ac901739s.

7. Brunkhorst R, Friedlaender F, Ferreirós N, Schwalm S, Koch A, Grammatikos G, et al. Alterations of the Ceramide Metabolism in the Peri-Infarct Cortex Are Independent of the Sphingomyelinase Pathway and Not Influenced by the Acid Sphingomyelinase Inhibitor Fluoxetine. Neural Plast. 2015; 2015: 503079. DOl: 10.1155/2015/503079.

8. Laaksonen R, Ekroos K, Sysi-Aho M, Hilvo M, Vihervaara T, Kauhanen D, et al. Plasma ceramides predict cardiovascular death in patients with stable coronary artery disease and acute coronary syndromes beyond LDL-cholesterol. Eur Heart J. 2016; 37 (25): 1967-76. DOI: 10.1093/eurheartj/ehw148.

9. Bligh EG, Dyer WJ. A rapid method of total lipid extraction and purification. Can J Biochem Physiol. 1959; 37 (8): 911-7.

10. Vasile VC, Meeusen JW, Medina Inojosa JR, Donato LJ, Scott CG, Hyun MS, et al. Ceramide Scores Predict Cardiovascular Risk in the Community. Arterioscler Thromb Vasc Biol. 2021; 41 (4): 1558-69. DOI: 10.1161/ATVBAHA.120.315530.

11. Li Q, Wang X, Pang J, Zhang Y, Zhang H, Xu Z, et al. Associations between plasma ceramides and mortality in patients with coronary artery disease. Atherosclerosis. 2020; 314: 77-83. DOI: 10.1016/j. atherosclerosis.2020.09.004

12. Poss AM, Maschek JA, Cox JE, Hauner BJ, Hopkins PN, Hunt SC, et al. Machine learning reveals serum sphingolipids as cholesterol- independent biomarkers of coronary artery disease. J Clin Invest. 2020; 130 (3): 1363-76. DOI: 10.1172/JCl131838.

13. You Q, Peng Q, Yu Z, Jin H, Zhang J, Sun W, et al. Plasma lipidomic analysis of sphingolipids in patients with large artery atherosclerosis cerebrovascular disease and cerebral small vessel disease. Biosci Rep. 2020; 40 (9): BSR20201519. DOI: 10.1042/ BSR20201519.

14. Han JS, Kim K, Jung $Y$, Lee JH, Namgung J, Lee HY, et al. Metabolic Alterations Associated with Atorvastatin/Fenofibric Acid Combination in Patients with Atherogenic Dyslipidaemia: A Randomized Trial for Comparison with Escalated-Dose Atorvastatin. Sci Rep. 2018; 8 (1): 14642. DOI: 10.1038/s41598018-33058-x.

15. Snowden SG, Grapov D, Settergren M, D'Alexandri FL, Haeggström JZ, Fiehn $\mathrm{O}$, et al. High-dose simvastatin exhibits enhanced lipid-lowering effects relative to simvastatin/ezetimibe combination therapy. Circ Cardiovasc Genet. 2014; 7 (6): 955-64. DOI: 10.1161/CIRCGENETICS.114.000606.

16. Ng TW, Ooi EM, Watts GF, Chan DC, Weir JM, Meikle PJ, et al. Dose-dependent effects of rosuvastatin on the plasma sphingolipidome and phospholipidome in the metabolic syndrome. J Clin Endocrinol Metab. 2014; 99 (11): E2335-40. DOI: 10.1210/jc.2014-1665.

17. Ng TW, Ooi EM, Watts GF, Chan DC, Meikle PJ, Barrett PH. Association of Plasma Ceramides and Sphingomyelin With VLDL apoB-100 Fractional Catabolic Rate Before and After Rosuvastatin Treatment. J Clin Endocrinol Metab. 2015; 100 (6): 2497-501. DOI: 10.1210/jc.2014-4348.

18. Feder S, Wiest R, Weiss TS, Aslanidis C, Schacherer D, Krautbauer S, et al. Proprotein convertase subtilisin/kexin type 9 (PCSK9) levels are not associated with severity of liver disease and are inversely related to cholesterol in a cohort of thirty eight patients with liver cirrhosis. Lipids Health Dis. 2021; 20 (1): 6. DOI: 10.1186/s12944-021-01431-x

19. Hilvo M, Simolin H, Metso J, Ruuth M, Öörni K, Jauhiainen M, et al. PCSK9 inhibition alters the lipidome of plasma and lipoprotein fractions. Atherosclerosis. 2018; 269: 159-65. DOI: 10.1016/j. atherosclerosis.2018.01.004.

20. Ye Q, Svatikova A, Meeusen JW, Kludtke EL, Kopecky SL. Effect of Proprotein Convertase Subtilisin/Kexin Type 9 Inhibitors on Plasma Ceramide Levels. Am J Cardiol. 2020; 128: 163-7. DOI: 10.1016/j.amjcard.2020.04.052

21. Tarasov K, Ekroos K, Suoniemi M, Kauhanen D, Sylvänne T, Hurme R, et al. Molecular lipids identify cardiovascular risk and are efficiently lowered by simvastatin and PCSK9 deficiency. J Clin Endocrinol Metab. 2014; 99 (1): E45-52. DOI: 10.1210/jc.20132559

22. Instrukcija k preparatu Pralujent. Dostupno po ssylke: http:// grls.rosminzdrav.ru/Grls_View_v2.aspx?routingGuid=e7885c432824-42ee-8673-f6221d7f2f5a\&t=. Russian. 
1. Bossard M, Latifi Y, Fabbri M, Kurmann R, Brinkert M, Wolfrum M, et al. Increasing Mortality From Premature Coronary Artery Disease in Women in the Rural United States. J Am Heart Assoc. 2020; 9 (9): e015334. DOI: 10.1161/JAHA.119.015334.

2. Mach F, Baigent C, Catapano AL, Koskinas KC, Casula M, Badimon L, et al; ESC Scientific Document Group. 2019 ESC/ EAS Guidelines for the management of dyslipidaemias: lipid modification to reduce cardiovascular risk. Eur Heart J. 2020; 41 (1): 111-88. DOI: 10.1093/eurheartj/ehz455.

3. Ference BA, Ginsberg HN, Graham I, Ray KK, Packard CJ, Bruckert E, et al. Low-density lipoproteins cause atherosclerotic cardiovascular disease. 1. Evidence from genetic, epidemiologic, and clinical studies. A consensus statement from the European Atherosclerosis Society Consensus Panel. Eur Heart J. 2017; 38 (32): 2459-72. DOI: 10.1093/eurheartj/ehx144.

4. Khera AV, Won HH, Peloso GM, Lawson KS, Bartz TM, Deng X, et al. Diagnostic Yield and Clinical Utility of Sequencing Familial Hypercholesterolemia Genes in Patients With Severe Hypercholesterolemia. J Am Coll Cardiol. 2016; 67 (22): 2578-89. DOI: 10.1016/j.jacc.2016.03.520.

5. Barenholz Y. Sphingomyelin and cholesterol: from membrane biophysics and rafts to potential medical applications. Subcell Biochem. 2004; 37: 167-215. DOI: 10.1007/978-1-4757-5806-1_5.

6. Manicke NE, Nefliu M, Wu C, Woods JW, Reiser V, Hendrickson RC, et al. Imaging of lipids in atheroma by desorption electrospray ionization mass spectrometry. Anal Chem. 2009; 81 (21): 8702-7. DOI: 10.1021/ac901739s.

7. Brunkhorst R, Friedlaender F, Ferreirós N, Schwalm S, Koch A, Grammatikos G, et al. Alterations of the Ceramide Metabolism in the Peri-Infarct Cortex Are Independent of the Sphingomyelinase Pathway and Not Influenced by the Acid Sphingomyelinase Inhibitor Fluoxetine. Neural Plast. 2015; 2015: 503079. DOI: $10.1155 / 2015 / 503079$.

8. Laaksonen R, Ekroos K, Sysi-Aho M, Hilvo M, Vihervaara T, Kauhanen D, et al. Plasma ceramides predict cardiovascular death in patients with stable coronary artery disease and acute coronary syndromes beyond LDL-cholesterol. Eur Heart J. 2016; 37 (25): 1967-76. DOI: 10.1093/eurhearti/ehw148.

9. Bligh EG, Dyer WJ. A rapid method of total lipid extraction and purification. Can J Biochem Physiol. 1959; 37 (8): 911-7.

10. Vasile VC, Meeusen JW, Medina Inojosa JR, Donato LJ, Scott CG, Hyun MS, et al. Ceramide Scores Predict Cardiovascular Risk in the Community. Arterioscler Thromb Vasc Biol. 2021; 41 (4): 1558-69. DOI: 10.1161/ATVBAHA.120.315530.

11. Li Q, Wang X, Pang J, Zhang Y, Zhang H, Xu Z, et al. Associations between plasma ceramides and mortality in patients with coronary artery disease. Atherosclerosis. 2020; 314: 77-83. DOI: 10.1016/j. atherosclerosis.2020.09.004.

12. Poss AM, Maschek JA, Cox JE, Hauner BJ, Hopkins PN, Hunt SC, et al. Machine learning reveals serum sphingolipids as cholesterol- independent biomarkers of coronary artery disease. J Clin Invest. 2020; 130 (3): 1363-76. DOI: 10.1172/JCl131838.

13. You Q, Peng Q, Yu Z, Jin H, Zhang J, Sun W, et al. Plasma lipidomic analysis of sphingolipids in patients with large artery atherosclerosis cerebrovascular disease and cerebral small vessel disease. Biosci Rep. 2020; 40 (9): BSR20201519. DOI: 10.1042/ BSR20201519.

14. Han JS, Kim K, Jung Y, Lee JH, Namgung J, Lee HY, et al. Metabolic Alterations Associated with Atorvastatin/Fenofibric Acid Combination in Patients with Atherogenic Dyslipidaemia: A Randomized Trial for Comparison with Escalated-Dose Atorvastatin. Sci Rep. 2018; 8 (1): 14642. DOI: 10.1038/s41598018-33058-x.

15. Snowden SG, Grapov D, Settergren M, D'Alexandri FL, Haeggström JZ, Fiehn O, et al. High-dose simvastatin exhibits enhanced lipid-lowering effects relative to simvastatin/ezetimibe combination therapy. Circ Cardiovasc Genet. 2014; 7 (6): 955-64. DOI: 10.1161/CIRCGENETICS.114.000606.

16. Ng TW, Ooi EM, Watts GF, Chan DC, Weir JM, Meikle PJ, et al. Dose-dependent effects of rosuvastatin on the plasma sphingolipidome and phospholipidome in the metabolic syndrome. J Clin Endocrinol Metab. 2014; 99 (11): E2335-40. DOI: 10.1210/jc.2014-1665.

17. Ng TW, Ooi EM, Watts GF, Chan DC, Meikle PJ, Barrett PH. Association of Plasma Ceramides and Sphingomyelin With VLDL apoB-100 Fractional Catabolic Rate Before and After Rosuvastatin Treatment. J Clin Endocrinol Metab. 2015; 100 (6): 2497-501. DOI: 10.1210/jc.2014-4348.

18. Feder S, Wiest R, Weiss TS, Aslanidis C, Schacherer D, Krautbauer $\mathrm{S}$, et al. Proprotein convertase subtilisin/kexin type 9 (PCSK9) levels are not associated with severity of liver disease and are inversely related to cholesterol in a cohort of thirty eight patients with liver cirrhosis. Lipids Health Dis. 2021; 20 (1): 6. DOI: 10.1186/s12944-021-01431-X.

19. Hilvo M, Simolin H, Metso J, Ruuth M, Öörni K, Jauhiainen M, et al. PCSK9 inhibition alters the lipidome of plasma and lipoprotein fractions. Atherosclerosis. 2018; 269: 159-65. DOI: 10.1016/j. atherosclerosis.2018.01.004.

20. Ye Q, Svatikova A, Meeusen JW, Kludtke EL, Kopecky SL. Effect of Proprotein Convertase Subtilisin/Kexin Type 9 Inhibitors on Plasma Ceramide Levels. Am J Cardiol. 2020; 128: 163-7. DOI: 10.1016/. .amjcard.2020.04.052.

21. Tarasov K, Ekroos K, Suoniemi M, Kauhanen D, Sylvänne T, Hurme R, et al. Molecular lipids identify cardiovascular risk and are efficiently lowered by simvastatin and PCSK9 deficiency. J Clin Endocrinol Metab. 2014; 99 (1): E45-52. DOI: 10.1210/jc.20132559.

22. Инструкция кпрепарату Пралуэнт. Доступно по ссылке: http:// grls.rosminzdrav.ru/Grls View v2.aspx?routingGuid=e7885c432824-42ee-8673-f6221d7f2f5a\&t= 